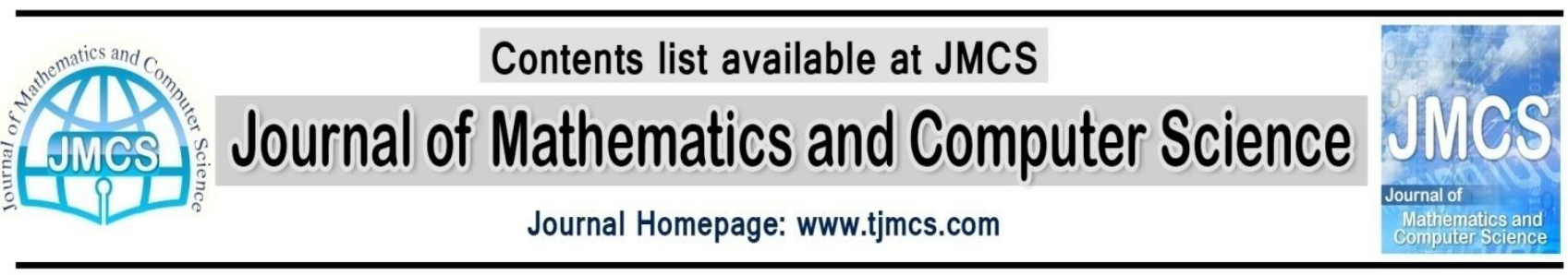

\title{
Numerical Solutions of MRLW Equation by a Fully Implicit Finite-Difference Scheme
}

\author{
Bilge İnan', Ahmet Refik Bahadir ${ }^{2}$ \\ ${ }^{1}$ Department of Mathematics, Faculty of Muallim Rifat Education, Kilis 7 Aralık \\ University, Kilis, Turkey \\ bilgeinan@kilis.edu.tr \\ ${ }^{2}$ Department of Mathematics, Faculty of Arts and Science, İnönü University, Malatya, \\ Turkey \\ refik.bahadir@inonu.edu.tr \\ Article history: \\ Received January 2015 \\ Accepted April 2015 \\ Available online April 2015

\section{Abstract} \\ In the present paper, a fully implicit finite difference method is introduced for the numerical \\ solution of the modified regularized long wave (MRLW) equation. The accuracy of the method is examined \\ by different problems of the MRLW equation. The results and comparisons with analytical and other \\ numerical invariants clearly show that results obtained using the fully implicit finite difference scheme are \\ precise and reliable.
}

Keywords: Modified regularized long wave equation; Fully implicit finite difference method; Solitary waves.

\section{Introduction}

The non-linear generalized regularized long wave (GRLW) equation has the form,

$$
\frac{\partial u}{\partial t}+\frac{\partial u}{\partial x}+\varepsilon u^{m} \frac{\partial u}{\partial x}-\mu \frac{\partial}{\partial t}\left(\frac{\partial^{2} u}{\partial x^{2}}\right)=0
$$

where $u$ is the wave amplitude, $\mu$ and $\varepsilon$ are positive parameter and $m$ is a positive integer. Physical boundary conditions require $u \rightarrow 0$ as $x \rightarrow \pm \infty$. This equation is very important in physics since it describes phenomena with weak nonlinearity and dispersion waves including nonlinear transverse waves in shallow water, ion acoustic and magnetohydrodynamic waves in plasma, and phonon packets in nonlinear cyrstals. Their solutions are kinds of solitary waves called solitons whose shapes are not affected by collision. A special case of Eq. (1) for $m=1$ is the regularized long wave (RLW) equation derived for long waves propagating with dispersion processes, has the following form, 


$$
\frac{\partial u}{\partial t}+\frac{\partial u}{\partial x}+\varepsilon u \frac{\partial u}{\partial x}-\mu \frac{\partial}{\partial t}\left(\frac{\partial^{2} u}{\partial x^{2}}\right)=0
$$

The equation was first obtained by Peregrine [1]. In this paper, we consider another special case of the generalized regularized long wave (GRLW) equation for $m=2$, called the modified regularized long wave (MRLW) equation given by

$$
\frac{\partial u}{\partial t}+\frac{\partial u}{\partial x}+\varepsilon u^{2} \frac{\partial u}{\partial x}-\mu \frac{\partial}{\partial t}\left(\frac{\partial^{2} u}{\partial x^{2}}\right)=0
$$

In literature, many numerical methods have been proposed and implemented for approximating solution of the MRLW equation. Khalifa et al. [2] solved the MRLW equation by collocation method using cubic B-splines finite element. Adomian decomposition method is applied for the modified regularized long wave equation by Khalifa et al. [3]. The homotopy perturbation method is used to implement the equation by Achouri et al. [4]. Raslan developed a new algorithm based on the collocation method to solve the MRLW equation [5]. Collocation methods using sextic B-splines have been developed for solving numerically the modified regularized long wave equation by Hassan et al. [6]. Raslan et al. [7] used Bspline finite element method to solve the equation. Dereli [8] solved the equation by using the meshless method based on collocation with well-known radial basis functions. Numerical scheme based on quartic B-spline collocation method is designed for the solution of MRLW equation by Haq et al. [9]. Dereli [10] used meshless kernel based method of lines by using radial basis functions for the modified regularized long wave equation. A homotopy analysis method employed to obtain approximate numerical solution of the equation by Khan et al. [11]. Karakoc et al. [12] solved the MRLW equation by a Petrov-Galerkin finite element method. Dag et al. [13] used a collocation method based on an extended cubic B-spline function for the numerical solution of the equation.

On the other hand, the fully implicit finite difference schemes are high-accuracy schemes for the numerical solution of the nonlinear problems. Bahadir employed the fully implicit finite difference method to compute an approximation to the solution of 1D [14] and 2D [15] Burgers' equations. İnan and Bahadir used the method for solving numerically the equal width wave equation [17].

In this paper, we develop a fully implicit finite difference scheme for solving the MRLW equation. To show efficiency and validity of the algorithm we apply this technique to several test examples and comparisons with the solutions obtained by other methods.

This paper is organized in four sections. In Section 2, we introduce the fully implicit finite difference scheme for the MRLW equation. Numerical examples are given in Section 3. Section 4 contains some conclusions.

\section{Fully implicit finite difference method for solving the MRLW equation}

The discretization is done by the finite differences with the implicit approach of solutions. Solution domain is discretized into cells described by the nodes set $\left(x_{i}, t_{n}\right)$ in which $x_{i}=i h,(i=0,1,2, \ldots, N)$ and $t_{n}=n k,(n=0,1,2, \ldots), \quad h$ is the spatial mesh size and $k$ is the time step.

Eq. (3) can be written in the following form:

$$
\frac{\partial u}{\partial t}+\frac{\partial u}{\partial x}+\frac{\varepsilon}{3} \frac{\partial u^{3}}{\partial x}-\mu \frac{\partial}{\partial t}\left(\frac{\partial^{2} u}{\partial x^{2}}\right)=0
$$


The derivatives of Eq. (4) can be approximated as

$$
\begin{aligned}
\frac{\partial u}{\partial t} & \cong \frac{U_{i}^{n+1}-U_{i}^{n}}{k} \\
\frac{\partial u}{\partial x} & \cong \frac{1}{2}\left[\frac{U_{i+1}^{n+1}-U_{i-1}^{n+1}}{2 h}+\frac{U_{i+1}^{n}-U_{i-1}^{n}}{2 h}\right] \\
\frac{\partial u^{3}}{\partial x} & \cong \frac{1}{2}\left[\frac{\left(U_{i+1}^{n+1}\right)^{3}-\left(U_{i-1}^{n+1}\right)^{3}}{2 h}+\frac{\left(U_{i+1}^{n}\right)^{3}-\left(U_{i-1}^{n}\right)^{3}}{2 h}\right] \\
\frac{\partial}{\partial t}\left(\frac{\partial^{2} u}{\partial x^{2}}\right) & \cong \frac{1}{k}\left[\frac{U_{i+1}^{n+1}-2 U_{i}^{n+1}+U_{i-1}^{n+1}}{h^{2}}-\frac{U_{i+1}^{n}-2 U_{i}^{n}+U_{i-1}^{n}}{h^{2}}\right] .
\end{aligned}
$$

Putting these approximations in (4) we get

$$
\begin{gathered}
\frac{U_{i}^{n+1}-U_{i}^{n}}{k}+\frac{1}{4 h}\left[U_{i+1}^{n+1}-U_{i-1}^{n+1}+U_{i+1}^{n}-U_{i-1}^{n}\right] \\
+\frac{\varepsilon}{12 h}\left[\left(U_{i+1}^{n+1}\right)^{3}-\left(U_{i-1}^{n+1}\right)^{3}+\left(U_{i+1}^{n}\right)^{3}-\left(U_{i-1}^{n}\right)^{3}\right] \\
-\frac{\mu}{k h^{2}}\left[U_{i+1}^{n+1}-2 U_{i}^{n+1}+U_{i-1}^{n+1}-U_{i+1}^{n}+2 U_{i}^{n}-U_{i-1}^{n}\right]=0
\end{gathered}
$$

which is valid for values of $i$ lying in the interval $1 \leq i \leq N-1$. Where $U_{i}^{n}$ denotes the finite difference approximation at the grid point $\left(x_{i}, t_{n}\right)$ to the exact solution $u(x, t)$. Eq. (5) is a system of nonlinear difference equations. Let us consider this nonlinear system of equations in the form

$$
\mathbf{F}(\mathbf{V})=\mathbf{0}
$$

where $\mathbf{F}=\left[f_{1}, f_{2}, \ldots, f_{N-1}\right]^{T}$ and $\mathbf{V}=\left[U_{1}^{n+1}, U_{2}^{n+1}, \ldots, U_{N-1}^{n+1}\right]^{T}$. Newton's method applied to Eq. (6) results in the following iteration:

1. Set $\mathbf{V}^{(0)}$, an initial guess.

2. For $m=0,1,2, \ldots$ until convergence do:

Solve $\boldsymbol{J}\left(\mathbf{V}^{(m)}\right) \boldsymbol{\delta}^{(m)}=-\boldsymbol{F}\left(\mathbf{V}^{(m)}\right) ;$

Set $\quad \mathbf{V}^{(m+1)}=\mathbf{V}^{(m)}+\boldsymbol{\delta}^{(m)}$ where $J\left(\mathbf{V}^{(m)}\right)$ is the Jacobian matrix which is evaluated analytically. The solution at the previous time-step is taken as the initial estimate. The Newton's iteration at each time-step is stopped when $\left\|\mathbf{F}\left(\mathbf{V}^{(m)}\right)\right\|_{\infty} \leq 10^{-5}$. The convergence is generally obtained in two or three iterations.

\section{Test Problems and Discussion}

In this section, some test problems have been considered to illustrate the performance of the method described in previous section.

The accuracy of the method is measured by using the $L_{2}$ and $L_{\infty}$ norms defined by 


$$
\begin{aligned}
& L_{2}=\|u-U\|_{2}=\left(h \sum_{i=0}^{N}\left|u_{i}-U_{i}\right|^{2}\right)^{\frac{1}{2}}, \\
& L_{\infty}=\|u-U\|_{\infty}=\max _{0 \leq i \leq N}\left|u_{i}-U_{i}\right| .
\end{aligned}
$$

Where $u$ and $U$ represent the exact and approximate solutions, respectively. We also examined our results by calculating the following three conserved quantities corresponding to mass, momentum and energy [16], respectively [10].

$$
\begin{aligned}
& I_{1}=\int_{-\infty}^{+\infty} u d x \cong h \sum_{i=1}^{N} U_{i}^{n}, \\
& I_{2}=\int_{-\infty}^{+\infty}\left(u^{2}+\mu\left(u_{x}\right)^{2}\right) d x \cong h \sum_{i=1}^{N}\left[\left(U_{i}^{n}\right)^{2}+\mu\left(\left(U_{x}\right)_{i}^{n}\right)^{2}\right] \\
& I_{3}=\int_{-\infty}^{+\infty}\left[u^{4}-\mu\left(u_{x}\right)^{2}\right] d x \cong h \sum_{i=1}^{N}\left[\left(U_{i}^{n}\right)^{4}-\mu\left(\left(U_{x}\right)_{i}^{n}\right)^{2}\right]
\end{aligned}
$$

To give a clear overview of the methodology the following examples will be discussed.

\subsection{Motion of single solitary wave}

We first model the motion of a single solitary wave of the MRLW equation. The solitary wave analytical solution of the MRLW equation (3) is

$$
u(x, t)=A \operatorname{sech}\left(p\left(x-(1+c) t-x^{*}\right)\right)
$$

where $p=\sqrt{c / \mu(1+c)}$. This solution corresponds to a solitary wave of amplitude $A=\sqrt{6 c / \varepsilon}$ and initially centered on the peak position $x^{*}$. The initial and boundary conditions are set to:

$u(x, 0)=A \operatorname{sech}\left(p\left(x-x^{*}\right)\right)$ and $u \rightarrow 0$ as $x \rightarrow \pm \infty$, respectively.

The analytical values of conservation quantities can be found as [2]

$$
I_{1}=\frac{\pi A}{p}, I_{2}=\frac{2 A^{2}}{p}+\frac{2 \mu p A^{2}}{3}, I_{3}=\frac{4 A^{2}}{3 p \varepsilon}\left(A^{2} \varepsilon-3 \mu p^{2}\right) .
$$

To allow comparison with the previous method parameters are taken as $\mu=1$ and $\varepsilon=6$. In the first case, we choose the parameters $c=1, h=0.2, k=0.025$ and $x^{*}=40$ over the solution domain $0 \leq x \leq 100$ . The analytical values for the invariants are $I_{1}=4.442883, I_{2}=3.299832$ and $I_{3}=1.414214$. The numerical solutions and $L_{2}$ and $L_{\infty}$ error norms are found for these parameters are presented in Table 1 . The growths of the invariants $I_{1}, I_{2}$ and $I_{3}$ from their initial values to $t=10$ are less than $5 \times 10^{-13}$, 
$1 \times 10^{-6}$ and $1 \times 10^{-5}$, respectively. The comparisons of numerical invariants with some previous results and analytical values of the invariants at $t=10$ for these parameters are given in Table 2. Figure 1 illustrates the motion of a single solitary wave for this case at different times. It is observed from Figure 1 that the single solitary wave moved to the right with constant amplitude and shape as time increases.

Table 1. Invariants and error norms for single solitary wave with $c=1$.

\begin{tabular}{|l|c|c|c|c|c|}
\hline \multicolumn{1}{|c|}{$t$} & $I_{1}$ & $I_{2}$ & $I_{3}$ & $L_{2}$ & $L_{\infty}$ \\
\hline 0 & 4.442883 & 3.299777 & 1.414268 & & \\
\hline 1 & 4.442883 & 3.299795 & 1.414236 & 0.0064435 & 0.0048730 \\
\hline 2 & 4.442883 & 3.299811 & 1.414206 & 0.0116747 & 0.0076591 \\
\hline 3 & 4.442883 & 3.299816 & 1.414188 & 0.0158906 & 0.0158906 \\
\hline 4 & 4.442883 & 3.299817 & 1.414176 & 0.0195888 & 0.0114470 \\
\hline 5 & 4.442883 & 3.299815 & 1.414167 & 0.0230392 & 0.0131584 \\
\hline 6 & 4.442883 & 3.299812 & 1.414160 & 0.0263702 & 0.0148681 \\
\hline 7 & 4.442883 & 3.299809 & 1.414153 & 0.0296452 & 0.0165691 \\
\hline 8 & 4.442883 & 3.299805 & 1.414147 & 0.0328959 & 0.0182659 \\
\hline 9 & 4.442883 & 3.299800 & 1.414142 & 0.0361388 & 0.0199612 \\
\hline 10 & 4.442883 & 3.299796 & 1.414136 & 0.0393822 & 0.0216564 \\
\hline
\end{tabular}

Table 2. The comparisons of results for single solitary wave at $t=10$.

\begin{tabular}{|l|l|l|l|}
\hline Method & \multicolumn{1}{|c|}{$I_{1}$} & \multicolumn{1}{c|}{$I_{2}$} & \multicolumn{1}{c|}{$I_{3}$} \\
\hline Analytical & 4.442883 & 3.299832 & 1.414214 \\
\hline Present & 4.442883 & 3.299796 & 1.414136 \\
\hline Khalifa et al. [2] & 4.44288 & 3.29983 & 1.41420 \\
\hline Raslan [5] $h=0.1, k=0.025)$ & 4.445176 & 3.302476 & 1.417411 \\
\hline Dereli [8] G & 4.44280 & 3.29957 & 1.41395 \\
\hline Karakoc et al. [12] & 4.4431758 & 3.3003023 & 1.4146927 \\
\hline
\end{tabular}

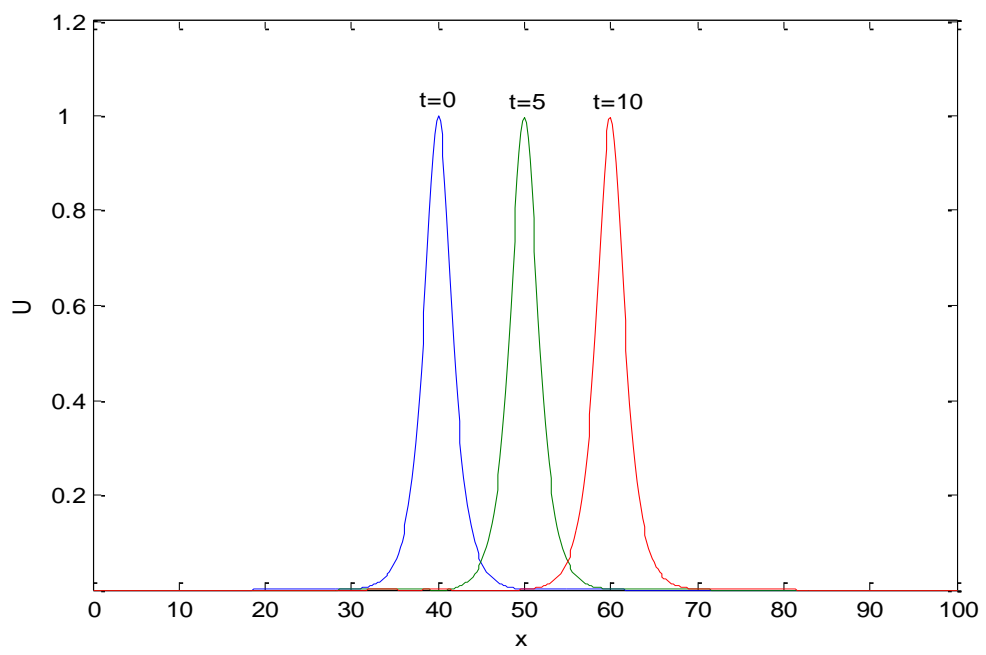

Figure 1: Single solitary wave of the equation with $c=1$. 
In the second case, the parameters are chosen $c=0.05, x^{*}=40$, with space step $h=0.1$, time step $k=0.1$ and with range $[0,80]$. The analytical values of the invariants are $I_{1}=3.219174$, $I_{2}=0.465532$ and $I_{3}=0.008001$. Invariants and error norms for a single solitary wave are presented in Table 3. It is observed that from Table 3, error norms are satisfactorily small. The growths in $I_{1}, I_{2}$ and $I_{3}$ from their initial values are $4 \times 10^{-5}, 6 \times 10^{-9}$ and $1 \times 10^{-8}$, respectively. These values indicate that our scheme provides high accuracy.

Table 3. Invariants and error norms for single solitary wave with $c=0.05$.

\begin{tabular}{|l|l|l|l|l|l|}
\hline \multicolumn{1}{|c|}{$t$} & \multicolumn{1}{c|}{$I_{1}$} & $I_{2}$ & $I_{3}$ & $L_{2}$ & $L_{\infty}$ \\
\hline 0 & 3.218504 & 0.465532 & 0.008001 & & \\
\hline 0.2 & 3.218503 & 0.465532 & 0.008001 & 0.000005 & 0.000002 \\
\hline 0.4 & 3.218501 & 0.465532 & 0.008001 & 0.000009 & 0.000004 \\
\hline 0.6 & 3.218499 & 0.465532 & 0.008001 & 0.000015 & 0.000007 \\
\hline 0.8 & 3.218494 & 0.465532 & 0.008001 & 0.000019 & 0.000010 \\
\hline 1.0 & 3.218489 & 0.465532 & 0.008001 & 0.000024 & 0.000013 \\
\hline 1.2 & 3.218483 & 0.465532 & 0.008001 & 0.000029 & 0.000016 \\
\hline 1.4 & 3.218475 & 0.465532 & 0.008001 & 0.000034 & 0.000019 \\
\hline 1.6 & 3.218466 & 0.465532 & 0.008001 & 0.000039 & 0.000022 \\
\hline 1.8 & 3.218456 & 0.465532 & 0.008001 & 0.000047 & 0.000026 \\
\hline 2.0 & 3.218444 & 0.465532 & 0.008001 & 0.000049 & 0.000029 \\
\hline
\end{tabular}

\subsection{Interaction of two solitary waves}

Secondly, the interaction process of two solitary waves traveling in the same direction is studied by using the initial condition

$$
u(x, 0)=A_{1} \operatorname{sech}\left(p_{1}\left(x-x_{1}^{*}\right)\right)+A_{2} \operatorname{sech}\left(p_{2}\left(x-x_{2}^{*}\right)\right)
$$

where $A_{j}=\sqrt{6 c_{j} / \varepsilon}, \quad p_{j}=\sqrt{c_{j} / \mu\left(1+c_{j}\right)}, j=1,2$ and the boundary conditions are $u \rightarrow 0$ as $x \rightarrow \pm \infty$. To allow comparison with the previous method parameters are taken as $\mu=1$ and $\varepsilon=6$.

The analytical values of the invariant quantities are [2]

$$
\begin{aligned}
& I_{1}=\sum_{i=1}^{2} \frac{\pi A_{i}}{p_{i}}, \\
& I_{2}=\sum_{i=1}^{2}\left(\frac{2 c_{i}}{p_{i}}+\frac{2 \mu p_{i} c_{i}}{3}\right), \\
& I_{3}=\sum_{i=1}^{2}\left(\frac{4 c_{i}^{2}}{3 p_{i}}-\frac{2 \mu p_{i} c_{i}}{3}\right) .
\end{aligned}
$$


Firstly, the parameters are taken as $c_{1}=4, c_{2}=1, x_{1}^{*}=25, x_{2}^{*}=55, h=0.1$ and $k=0.025$ over the solution domain $0 \leq x \leq 250$, then the amplitudes are in ratio 2:1, where $A_{1}=2 A_{2}$. The results are tabulated in Table 4. The analytical values of the invariants are $I_{1}=11.467698, I_{2}=14.629243$ and $I_{3}=22.880466$ and the growth of invariants $I_{1}, I_{2}$ and $I_{3}$ are $1 \times 10^{-7}, 0.025137$ and 0.113372 , respectively. Comparisons of the invariants for this case are documented in Table 5. Figure 2 shows the interaction of two solitary waves for these parameters at different time levels. It can be seen from Figure 2 that the larger solitary wave has passed the smaller solitary wave as time increases. Then, two solitary waves regain their original shape after the interaction.

Table 4. Invariants for interaction of two solitary waves.

\begin{tabular}{|l|l|l|l|}
\hline \multicolumn{1}{|c|}{$t$} & \multicolumn{1}{c|}{$I_{1}$} & \multicolumn{1}{c|}{$I_{2}$} & $I_{3}$ \\
\hline 0 & 11.467698 & 14.629194 & 22.880514 \\
\hline 1 & 11.467698 & 14.626621 & 22.870153 \\
\hline 2 & 11.467698 & 14.624007 & 22.859699 \\
\hline 3 & 11.467698 & 14.621398 & 22.849280 \\
\hline 4 & 11.467698 & 14.618792 & 22.838876 \\
\hline 5 & 11.467698 & 14.616187 & 22.828464 \\
\hline 6 & 11.467698 & 14.613584 & 22.818009 \\
\hline 7 & 11.467698 & 14.610848 & 22.806149 \\
\hline 8 & 11.467698 & 14.607107 & 22.784129 \\
\hline 9 & 11.467698 & 14.598231 & 22.715753 \\
\hline 10 & 11.467698 & 14.604057 & 22.767142 \\
\hline
\end{tabular}

Table 5. The comparisons of results for interaction of two solitary waves at $t=10$.

\begin{tabular}{|l|c|c|c|}
\hline Method & $I_{1}$ & $I_{2}$ & $I_{3}$ \\
\hline Analytical & 11.467698 & 14.629243 & 22.880466 \\
\hline Present & 11.467698 & 14.604057 & 22.767142 \\
\hline Raslan et al. [7] $(h=0.2, k=0.025)$ & 11.460050 & 14.608160 & 22.842790 \\
\hline Haq et al. [9] $(h=0.2, k=0.025)$ & 11.467700 & 14.603687 & 22.771773 \\
\hline
\end{tabular}



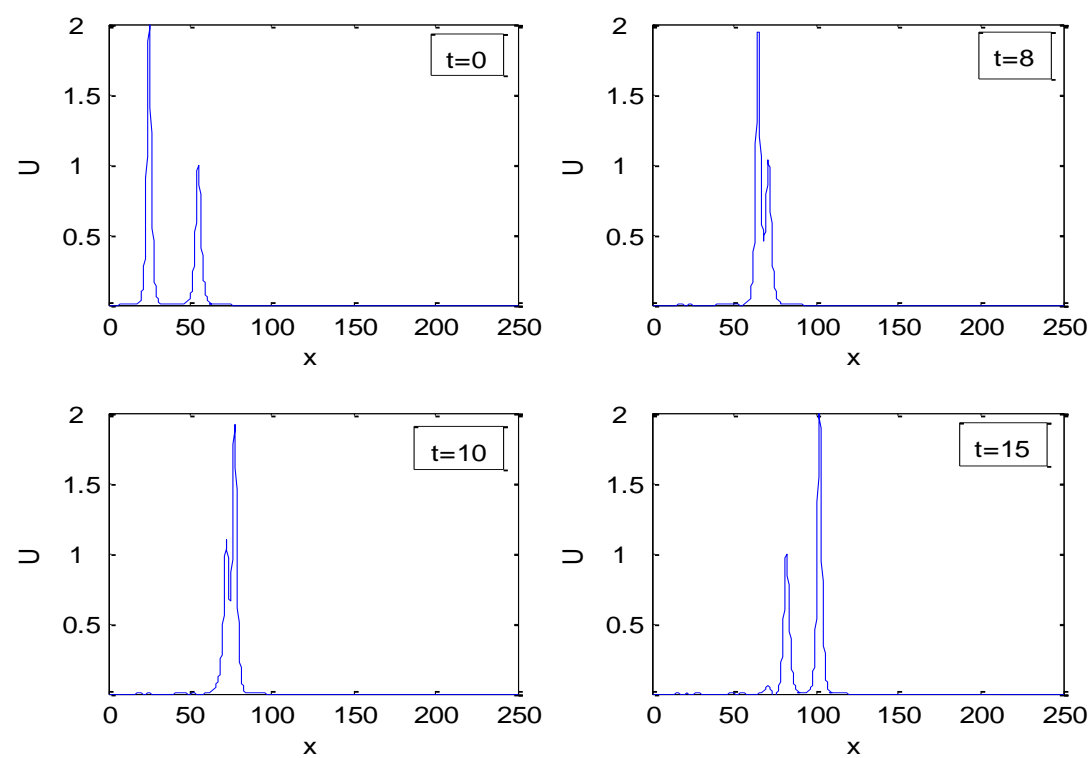

Figure 2: Interaction of two solitary waves at selected times.

Secondly, we chose $c_{1}=1, c_{2}=0.5, x_{1}^{*}=10, x_{2}^{*}=40, h=0.2$ and $k=0.05$ over the solution domain $0 \leq x \leq 100$. Invariants for interaction of two solitary waves are given in Table 6 . The analytical values of the invariants are $I_{1}=8.29053243, I_{2}=5.22433254$ and $I_{3}=1.79911374$. The growths of invariants $I_{1}, I_{2}$ and $I_{3}$ are $1 \times 10^{-3}, 6 \times 10^{-5}$ and $8 \times 10^{-5}$, respectively.

Table 6. Invariants for interaction of two solitary waves.

\begin{tabular}{|l|l|l|l|}
\hline \multicolumn{1}{|c|}{$t$} & \multicolumn{1}{c|}{$I_{1}$} & \multicolumn{1}{c|}{$I_{2}$} & $I_{3}$ \\
\hline 0 & 8.287956 & 5.224284 & 1.799162 \\
\hline 2 & 8.292722 & 5.224238 & 1.799022 \\
\hline 4 & 8.294685 & 5.224171 & 1.798906 \\
\hline 6 & 8.296623 & 5.224095 & 1.798812 \\
\hline 8 & 8.298449 & 5.224015 & 1.798727 \\
\hline 10 & 8.299991 & 5.223934 & 1.798644 \\
\hline 12 & 8.301186 & 5.223852 & 1.798562 \\
\hline 14 & 8.302087 & 5.223768 & 1.798483 \\
\hline 16 & 8.302801 & 5.223699 & 1.798376 \\
\hline 18 & 8.303417 & 5.223601 & 1.798331 \\
\hline 20 & 8.303961 & 5.223505 & 1.798281 \\
\hline
\end{tabular}

\subsection{Interaction of three solitary waves}

Thirdly, the interaction process of three solitary waves traveling in the same direction is studied by using the initial condition

$$
\begin{aligned}
u(x, 0) & =A_{1} \operatorname{sech}\left(p_{1}\left(x-x_{1}^{*}\right)\right)+A_{2} \operatorname{sech}\left(p_{2}\left(x-x_{2}^{*}\right)\right) \\
& +A_{3} \operatorname{sech}\left(p_{3}\left(x-x_{3}^{*}\right)\right)
\end{aligned}
$$


where $A_{j}=\sqrt{6 c_{j} / \varepsilon}, \quad p_{j}=\sqrt{c_{j} / \mu\left(1+c_{j}\right)}, j=1,2,3$ and the boundary conditions are $u \rightarrow 0$ as $x \rightarrow \pm \infty$. To allow comparison with the previous method parameters are taken as $\mu=1$ and $\varepsilon=6$. The analytical values of the invariant quantities are [2]

$$
\begin{aligned}
& I_{1}=\sum_{i=1}^{3} \frac{\pi A_{i}}{p_{i}} \\
& I_{2}=\sum_{i=1}^{3}\left(\frac{2 c_{i}}{p_{i}}+\frac{2 \mu p_{i} c_{i}}{3}\right), \\
& I_{3}=\sum_{i=1}^{3}\left(\frac{4 c_{i}^{2}}{3 p_{i}}-\frac{2 \mu p_{i} c_{i}}{3}\right) .
\end{aligned}
$$

In this case, following parameters are used: $c_{1}=4, c_{2}=1, c_{3}=0.25, x_{1}^{*}=15, x_{2}^{*}=45$, $x_{3}^{*}=60, h=0.2$ and $k=0.025$ over the solution domain $0 \leq x \leq 250$. Thus the amplitudes are in the ratio 4:2:1 where $A_{1}=2 A_{2}=4 A_{3}$. The analytical values of invariants for the interaction of three waves are $I_{1}=14.980105, I_{2}=15.821812$ and $I_{3}=22.992270$. Numerical values of the invariants are obtained by fully implicit finite difference method are recorded in Table 7. The growths in $I_{1}, I_{2}$ and $I_{3}$ are $4 \times 10^{-5}, 0.014989$ and 0.146276 , respectively. The comparison of invariants for these parameters are given in Table 8 . Figure 3 presents interaction of these solitary waves to $t=25$. Figure 3 shows that the largest solitary wave has passed the smallest solitary wave as time increases.

Table 7. Invariants for interaction of three solitary waves.

\begin{tabular}{|l|c|l|l|}
\hline \multicolumn{1}{|c|}{$t$} & $I_{1}$ & $I_{2}$ & $I_{3}$ \\
\hline 0 & 14.980098 & 15.836732 & 23.008932 \\
\hline 1 & 14.980112 & 15.834559 & 22.997699 \\
\hline 2 & 14.980115 & 15.832109 & 22.987443 \\
\hline 3 & 14.980119 & 15.829650 & 22.977288 \\
\hline 4 & 14.980122 & 15.827206 & 22.967134 \\
\hline 5 & 14.980126 & 15.824793 & 22.956927 \\
\hline 6 & 14.980129 & 15.822343 & 22.946991 \\
\hline 7 & 14.980133 & 15.820419 & 22.934121 \\
\hline 8 & 14.980136 & 15.819951 & 22.908805 \\
\hline 9 & 14.980139 & 15.820619 & 22.832402 \\
\hline 10 & 14.980141 & 15.821743 & 22.862656 \\
\hline
\end{tabular}


Table 8. The comparisons of results for interaction of three solitary waves $t=10$.

\begin{tabular}{|l|l|l|l|}
\hline Method & \multicolumn{1}{|c|}{$I_{1}$} & \multicolumn{1}{c|}{$I_{2}$} & \multicolumn{1}{c|}{$I_{3}$} \\
\hline Analytical & 14.980105 & 15.821812 & 22.992269 \\
\hline Present & 14.980141 & 15.821743 & 22.862656 \\
\hline Khalifa et al. [2] & 13.6891 & 15.6514 & 22.8388 \\
\hline Raslan et al. [7] & 14.930390 & 15.822500 & 22.964190 \\
\hline Dereli [8] G $(h=0.5, k=0.01)$ & 14.9794 & 15.8049 & 22.8703 \\
\hline
\end{tabular}
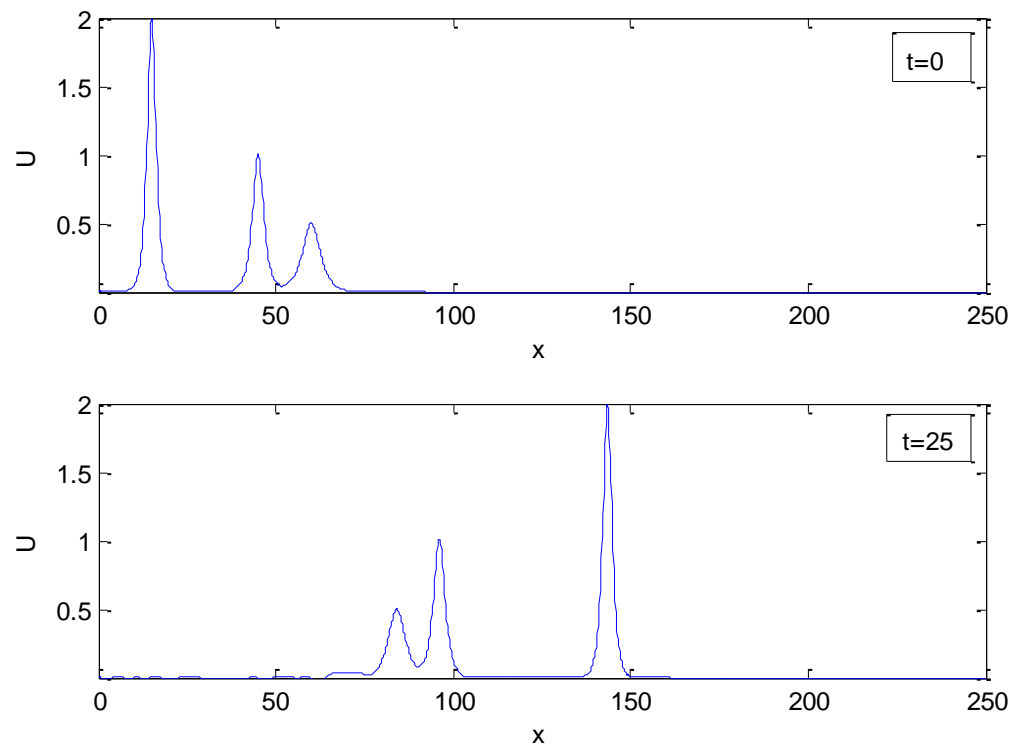

Figure 3: Interaction of three solitary waves at selected times.

\subsection{The Maxwellian initial condition}

Finally, we use the initial condition

$$
u(x, 0)=\exp \left(-(x-7)^{2}\right)
$$

and the boundary conditions are $u \rightarrow 0$ as $x \rightarrow \pm \infty$ to analyse the evolution of an Maxwellian pulse into solitary waves.

In this test, we study for values of $\mu=0.1, \mu=0.04, \mu=0.015, \mu=0.01$ and $\varepsilon=6$ through the interval $0 \leq x \leq 100$.

Table 9 gives the invariants obtained from the present method for $h=0.1, k=0.001$. The growth of $I_{1}, I_{2}, I_{3}$ are given in Table 10. Figure 4 shows wave profiles for the Maxwellian initial condition at $t=15$ for $0 \leq x \leq 100$. This figure contains figures for $\mu=0.1, \mu=0.04, \mu=0.015$ and $\mu=0.01$, respectively. As can be seen from Figure $4, \mu$ is reduced more and more solitary waves are formed, as expected. 
Table 9. Invariants for the Maxwellian initial condition.

\begin{tabular}{|c|c|c|c|c|c|c|c|c|}
\hline $\mathrm{t}$ & $\mu$ & $I_{1}$ & $I_{2}$ & $I_{3}$ & $\mu$ & $I_{1}$ & $I_{2}$ & $I_{3}$ \\
\hline 0.01 & \multirow[t]{3}{*}{0.1} & 1.772454 & 1.378632 & 0.760909 & \multirow{3}{*}{0.015} & 1.772454 & 1.272108 & 0.867433 \\
\hline 0.03 & & 1.772454 & 1.378623 & 0.760918 & & 1.772454 & 1.272078 & 0.867463 \\
\hline 0.05 & & 1.772454 & 1.378606 & 0.760936 & & 1.772454 & 1.272015 & 0.867526 \\
\hline 0.01 & \multirow[t]{3}{*}{0.04} & 1.772454 & 1.303440 & 0.836102 & \multirow[t]{3}{*}{0.01} & 1.772454 & 1.265842 & 0.873699 \\
\hline 0.03 & & 1.772454 & 1.303420 & 0.836121 & & 1.772454 & 1.265808 & 0.873734 \\
\hline 0.05 & & 1.772454 & 1.303382 & 0.836159 & & 1.772454 & 1.265737 & 0.873805 \\
\hline
\end{tabular}

Table 10. The growth of invariants for the Maxwellian initial condition.

\begin{tabular}{|l|l|l|l|}
\hline$\mu$ & \multicolumn{1}{|c|}{$I_{1}$} & \multicolumn{1}{|c|}{$I_{2}$} & $I_{3}$ \\
\hline 0.1 & $1 \times 10^{-1^{6}}$ & $2 \times 10^{-5}$ & $2 \times 10^{-5}$ \\
\hline 0.04 & $2 \times 10^{-1^{5}}$ & $5 \times 10^{-5}$ & $5 \times 10^{-5}$ \\
\hline 0.015 & $1 \times 10^{-1^{5}}$ & $1 \times 10^{-5}$ & $1 \times 10^{-5}$ \\
\hline 0.01 & $1 \times 10^{-1^{5}}$ & $1 \times 10^{-4}$ & $1 \times 10^{-4}$ \\
\hline
\end{tabular}
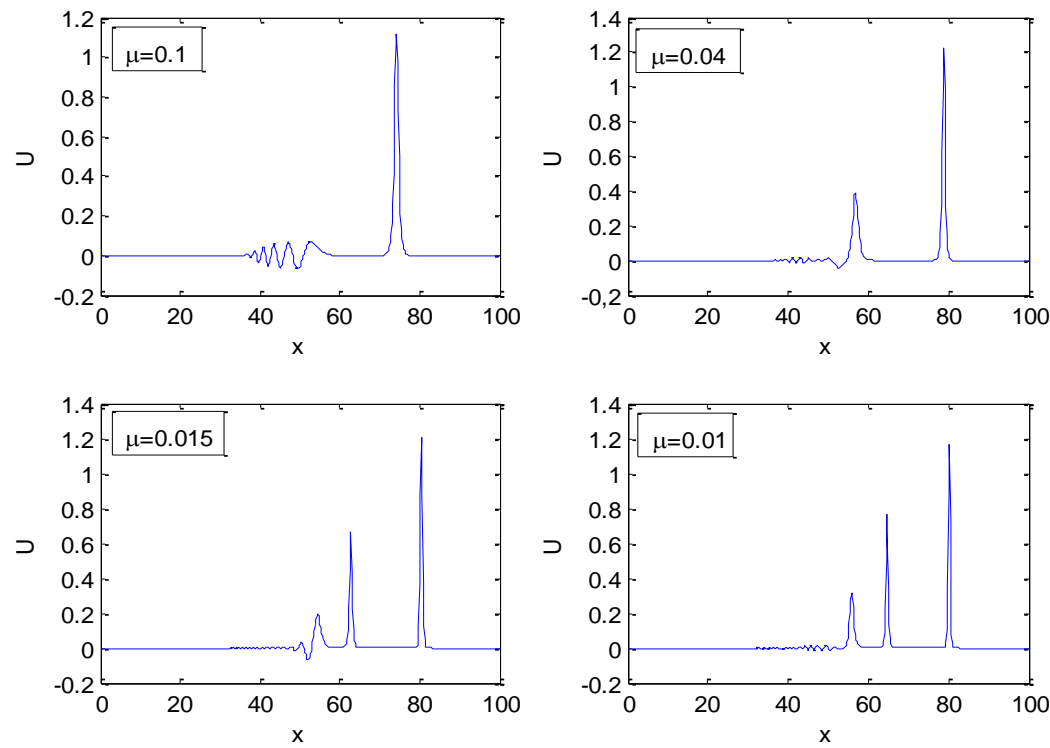

Figure 4: Maxwellian initial condition for $k=0.025, h=0.2$ at $t=15$.

\section{Conclusion}

In this paper, a fully implicit finite difference method has been successfully applied to finding the solution of the MRLW equation. Numerical tests for single solitary wave, interaction of two solitary waves, interaction of three solitary waves and Maxwellian initial condition are considered by the fully implicit finite difference method. The numerical results demonstrate that the present method is quite accurate and readily implemented in the solution of the MRLW equation. 


\section{References}

[1] D. H. Peregrine, “Calculations of development of an undular bore”, J. Fluid Mech. 25 (1966) 321330.

[2] A. K. Khalifa, K. R. Raslan, H. M. Alzubaidi, "A collocation method with cubic B-splines for solving the MRLW equation", J. Comput. Appl. Math. 212 (2008) 406-418.

[3] A. K. Khalifa, K. R. Raslan, H. M. Alzubaidi, "Numerical study using ADM for the modified regularized long wave equation", Appl. Math. Modelling, 32 (2008) 2962-2972.

[4] T. Achouri, K. Omrani, "Application of the homotopy perturbation method to the modified regularized long-wave equation", Numer. Meth. Part. D. E. 26 (2009) 399-411.

[5] K. R. Raslan, "Numerical study of the modified regularized long wave (MRLW) equation", Chaos, Solitons and Fract. 42 (2009) 1845-1853.

[6] S. M. Hassan, D. G. Alamery, "B-splines collocation algorithms for solving numerically the MRLW equation", Int. J. Nonlin. Sci. 8 (2009) 131-140.

[7] K. R. Raslan, T. S. El-Danaf, "Solitary waves solutions of the MRLW eqution using quintic Bsplines", J. King Saud University (Science) 22 (2010) 161-166.

[8] Y. Dereli, "Solitary wave solutions of the MRLW equation using radial basis functions", Numer. Meth. Part. D. E. 28 (2010) 235-247.

[9] F. Haq, S. Islam, I. A. Tirmizi, "A numerical technique for solution of the MRLW equation using quartic B-splines", Appl. Math. Modelling, 34 (2010) 4151-4160.

[10] Y. Dereli, "Numerical solutions of the MRLW equation using meshless kernel based method of lines", Int. J. Nonlin. Sci. 13 (2012) 28-38.

[11] Y. Khan, R. Taghipour, M. Falahian, A. Nikkar, "A new approach to modified regularized long wave equation", Neural Comput. \& Applic. Doi 10.1007/s00521-012-1077-0.

[12] S. B. G. Karakoc, T. Geyikli, "Petrov-Galerkin finite element method for solving the MRLW equation", Math. Sciences, (2013) 7:25.

[13] İ. Dağ, D. Irk, M. Sar1, "The extended cubic B-spline algorithm for a modified regularized long wave equation", Chin. Phys. B, 22 (2013) 040207.

[14] A. R. Bahadır, "Numerical solution for one-dimensional Burgers' equation using a fully implicit finite-difference method", Int. J. Appl. Math. 1 (1999) 897-909.

[15] A. R. Bahadır, "A fully implicit finite-difference scheme for two-dimensional Burgers' equations", Appl. Math. Comput. 137 (2003) 131-137.

[16] P.J. Olver, "Euler operators and conservation laws of the BBM equation", Math. Proc. Cambridge Philos. Soc. 85 (1979) 143-160.

[17] B. İnan, A. R. Bahadır, "A numerical solution of the equal width wave equation using a fully implicit finite difference method", Turkish Journal of Mathematics \& Computer Science, Article ID 20140037, (2014) 14 pages. 